\title{
La interculturalidad en el aprendizaje y la comunicación del diseño social
}

\author{
Interculturality in learning and social design communication \\ A interculturalidade na aprendizagem e na comunicação do desenho social
}

DOI: http://dx.doi.org/10.21803\%2Fpenamer.11.21.523

Ana Georgina Navarro Sarmiento https://orcid.org/0000-0002-2234-3157

Claudia Padilla Camberos https://orcid.org/0000-0003-4330-6272

Julio Alejandro Santillán Becerra https://orcid.org/0000-0001-6781-4314

\section{¿Cómo citar este artículo?}

Navarro, A., Padilla, C. \& Santillán J. (2018). La interculturalidad en el aprendizaje y la comunicación del diseño social. Pensamiento Americano, 11(21), 45-59.

DOI:http://dx.doi.org/10.21803\%2Fpenamer.11.21.523

\section{Resumen}

Se analizarán las etapas del proceso de diseño social desde el punto de vista académico y la importancia de cómo influye la comunicación intercultural en el desarrollo del país en donde éste se da y la relación que se pudiese lograr hacía con los demás.

El hablar del proceso del diseño social en un ambiente universitario nos puede ubicar en un área de interés en la que se plantean las formas de cómo se piensa, cómo se hace y cómo se comunica el diseño.

El diseño social juega actualmente un papel muy importante en donde se involucra a la sociedad a partir de las necesidades y deseos expresados por esta, en donde se contempla que se optimice los recursos y las capacidades, y que se ve más como un sistema social que productivo y en donde el objetivo final sería que se lograra una fácil, útil y práctica comunicación global.

PALABRAS CLAVES: Diseño social, aprendizaje y comunicación intercultural.

\section{Abstract}

Will analyze the stages of the social design process from the academic point of view and the importance of how intercultural communication influences the development of the country where it occurs and the relationship that could be achieved with others.

Talking about the process of social design in a university environment can place us in an area of interest in which the ways of how people think, how they are done and how design is communicated are presented.

Social design currently plays a very important role in which society is involved based on the needs and desires expressed by it, where it is contemplated that resources and capacities are optimized, and that it is seen more as a social system than productive and where the ultimate goal would be to achieve an easy, useful and practical global communication.

KEYWORDS: State, public policies, human rights, rights-based approach 


\section{Resumo}

Nesta pesquisa se analisarão as etapas do processo de desenho social desde o ponto-de-vista acadêmico e a importância de como influencia a comunicação intercultural no desenvolvimento do país onde se dá, e a relação que se pode conseguir aos demais.

Falar do processo de desenho social num ambiente universitário nos pode localizar em uma área de interesse nas quais se projetam as formas de como se pensa, como se faz e como se comunica o desenho.

O desenho social representa atualmente um papel muito importante onde se envolve a sociedade a partir das necessidades e desejos expressos pela mesma, onde se contempla que se otimiza os recursos e as capacidades, e que se veja mais como um sistema social que produtivo, e onde o objetivo final seria que se conseguisse uma fácil, útil e prática comunicação global.

PALAVRAS CHAVE: Desenho social, aprendizagem e comunicação intercultural.

\section{Perfil}

Licenciatura en Diseño de Interiores y Ambientación por la Universidad de Guadalajara. Maestría en Metodología de la enseñanza por el Instituto Mexicano de Pedagogía en Guadalajara Jalisco México. Doctorado en Ciencias de la Educación. por la Universidad Santander, Jalisco-México. Profesor de tiempo completo Universidad De Guadalajara.

georgina.navarrro@cutonala.udg.mx

\section{Perfil}

Química Farmacobióloga, maestría en Química Clínica, la Licenciatura en Psicología, con un Diplomado en Terapia Cognitivo Conductual, Universidad de Guadalajara. Doctorado en Competencias Educativas, en el Instituto Pedagógico de Estudios Superiores del Estado de Jalisco. Profesor de Tiempo Completo en el Departamento de Justicia Alternativa, Ciencias Forenses y Disciplinas Auxiliares al Derecho. Centro Universitario De Tonalá. Universidad De Guadalajara Jalisco, México claudiapadi@hotmail.com

\section{Perfil}

Licenciatura en turismo, Maestría en Planeación de la educación superior, Universidad de Guadalajara. Doctor en derecho, Instituto de Altos Estudios Jurídicos. Profesor investigador en la Universidad de Guadalajara. (México) julio.santillan@cutonala.udg.mx

\section{Ana Georgina Navarro Sarmiento}

Universidad de Guadalajara, México

\section{Claudia \\ Padilla Camberos}

Universidad de Guadalajara, México

\section{Julio Alejandro \\ Santillán Becerra}

Universidad de Guadalajara, México 


\section{El Diseño}

N os adentraremos al conocimiento y análisis de las diferentes ideas y pensamientos que algunos autores tienen en relación con el concepto de diseño.

Para Aquiles Gay \& Lidia Samar (2007) la palabra diseño hace referencia a la preconcepción sistematizada de la forma y las demás características del producto, teniendo en cuenta los aspectos sociales, tecnológicos, estéticos, psicológicos, anatómicos, fisiológicos, etc.

Para Guillan Scott, (1969) diseño es toda acción creadora que cumple una finalidad: que es la de suplir una necesidad, él menciona que el diseño es algo inherente en el ser humano, que es indispensable para la existencia del arte, que es el primer paso de todo proceso creativo; el inicio de la búsqueda de la solución a cualquier dificultad, además una característica básica del ser humano y que de alguna forma afecta a todas las personas con sus detalles esenciales, ya que con ellos se deciden actividades, usos, costumbres, que se busca el goce y regocijo estético y muchos lineamientos del accionar de todos.

Wucius Wong (1995) menciona en su libro "Fundamentos del Diseño" que la disciplina del diseño es más que algún tipo de esfuerzo dedicado al embellecimiento de la apariencia exterior de las cosas, que solo el embellecimiento de las cosas es una parte del diseño, mencionando que el diseño no es un adorno, nos sugiere mirar a nuestro alrededor y veamos al diseño como un proceso de creación visual con un propósito. Comenta en pocas palabras que un buen diseño es la mejor expresión visual de la esencia de "algo", ya sea esto un mensaje o un producto y que, para hacerlo fiel y eficazmente, el diseñador debe buscar la mejor forma posible para que ese "algo" sea conformado, fabricado, distribuido, usado y relacionado con su ambiente.

Los anteriores autores definen el concepto de "diseño" como la actividad o acción de un proceso creativo que busca dar solución a algún problema de diseño en particular; en donde en primera instancia mencionan que se deben de tomar en cuenta los aspectos sociales, tecnológicos, estéticos, funcionales, emocionales y físicos, entre otros. Estas definiciones coinciden con la actividad que el profesor de la materia de diseño desarrolla en el aula durante el proceso de enseñanza aprendizaje con los estudiantes; proponemos entonces que el profesor se dé a la tarea de ser creativo, de implementar actividades que promuevan que los estudiantes propongan soluciones más globales e individualistas en su propio proceso académico del aula, ya que los estudiantes de diseño crean su propia solución en relación a la solución de una problemática planteada con anterioridad, misma que deberá estar basada en algún problema específico en donde intervenga la parte intercultural en sus diferentes disciplinas, involucrado en las necesidades sociales a nivel global.

\section{Aspectos históricos de la disciplina llamada diseño}

Históricamente el diseño ha ido evolucionando en sus métodos de creación, así como en el proceso de enseñanza por las condiciones sociales y las demandas culturales de cada época.

Aquiles Gay \& Lidia Samar (2007) comentan que el proceso de preconcepción es clave en la producción industrial pues para ellos es imposible fabricar industrialmente un objeto sin antes haber definido con precisión sus características físicas y de producción. Esto es justamente lo que los profesores deben tener en consideración cuando dirigen un proceso de diseño: tomando en cuento lo que la sociedad demanda en algún momento, en el cual no 
solo deben abordar aspectos estéticos, sino además manejar un enfoque sustentable para el ambiente.

En función de lo planteado y en relación con la historia del diseño industrial, se ha considerado como punto germinal "la Gran Exposición Internacional de Londres de 1857" (The Greaí Exibition of the Works of Industry ofall Nations, 1857), la primera exposición en donde le presentaron al gran público productos industriales, muchos de los cuales denotaban una falta de armonía entre forma y decoración. Recordándonos que en esa época se apelaba a la decoración como factor de "embellecimiento" del producto, lo que provocó un cuestionamiento a la calidad estética de la producción industrial, y consecuentemente surgió como problema la necesidad de ocuparse del diseño de los objetos antes de producirlos.

Se ha podido observar que la disciplina del diseño ha ido evolucionando según las condiciones político-sociales, ya que en sus inicios los objetos de diseño tenían que ser útiles y el usuario se tenía que adaptar a ellos; posteriormente tomaron en cuenta la opinión del usuario y con esto produjeron la combinación de utilidad y estética, al pasar de los años podemos darnos cuenta que el cambio está en la sustentabilidad, es decir en la responsabilidad social.

La disciplina del diseño, así como la acción de la misma ha ido progresando al incluir como segunda palabra su especialidad. Es decir cuando dicen que un objeto de diseño es un producto útil, estético y que además presenta un proceso industrial, arquitectónico, textil, urbano, artesanal, o de espacios interiores, ya están hablando de algunas especialidades que se han desarrollado a la par con el avance del diseño y las industrias o procesos específicos, el concepto de diseño social es relativamente nuevo y debemos aprovechar esa oportuni- dad para poder amalgamar los conocimientos para logar soluciones de diseño que ayuden a elevar la calidad de vida.

César Vargas, \& Elizabeth Oguri (2013) consideran que el diseño industrial busca crear o modificar objetos o ideas para hacerlos útiles, prácticos y atractivos visualmente, con la intención de satisfacer las necesidades del ser humano, adaptando los objetos e ideas no sólo en su forma sino también en cuanto a las funciones de éste, su concepto, su contexto y su escala, busca lograr un producto final que sea innovador. Esta definición hace referencia del diseño para la industria y el comercio.

Cuando se habla de la historia del diseño se habla de la Bauhaus. Nos limitaremos a comentar los aspectos más importantes de su filosofía, los cambios de sus paradigmas académicos, así como lo más relevante de sus procesos de enseñanza en relación al usuario como destinatario final en un contexto social.

Cembellín (2004) menciona en su artículo BAUHAUS "La escuela que unió arte y técnica", que ésta escuela marcó un hito artístico, filosófico e industrial que todavía se percibe en la actualidad en diferentes universidades, el empeño de lograr la síntesis del artey la técnica trajo una nueva forma de pensar y ver el mundo que atisbó el futuro de una floreciente industria, esta tendencia fue un revulsivo para el diseño industrial, introduciéndolo en la vida cotidiana. El claro enfoque funcionalista proporcionó los cimientos del diseño industrial y del movimiento moderno, pero su influencia no se ha limitado solo al diseño, sino que las bases de su sistema educativo se importarían y aplicarían en las futuras escuelas de diseño y arquitectura de todo el mundo.

La escuela de la Bauhaus (1919-1933) marcaba una nueva unidad entre el arte y la artesanía con su manifiesto: "Artistas y Artesanos de- 
ben levantar juntos la construcción del futuro", con ésta, idea la escuela comenzó su actividad, con la única idea de reconciliar a artistas y artesanos que hasta ese momento eran independientes para adoptar el trabajo en equipo fusionando las distintas artes. Esta fusión ha sido aplicada en el Centro Universitario de Tonalá, en la carrera de Diseño de Artesanías, donde artistas, profesionistas del diseño y artesanos imparten clases, aportando una visión conjunta con visión al futuro global.

En la Bauhaus, el plan de estudios consistió en un curso preparatorio, en el que los estudiantes aprendían los conceptos básicos del diseño y de la teoría del color, cuando los alumnos aprobaban estos cursos eran aceptados en los talleres, en donde ellos se especializaban en algún tipo de oficio artesanal; en relación a la implementación de los talleres bastó con el entusiasmo de los antiguos encargados y maestros de los mismos que llegaron a acuerdos y así iniciaron con los talleres de encuadernación e impresión gráfica; el taller de textil poco tiempo después funcionaría junto con los de carpintería, el de pintura en vidrio y el de alfarería.

A mediados del año 1921 el artista holandés Theo van Doesbrurg, se instaló como profesor en Weimar impartiendo sus cursos al margen de la Bauhaus, su influencia marcó un radical cambio hacia una concepción del diseño más técnica y constructiva. El programa de estudios adquirió entonces un carácter más experimental y de investigación. Desde el inicio de la Licenciatura en Diseño de Artesanías en CUTonalá en el 2012, Este sistema de aprendizaje basado en proyectos, se ha planteado y ha funcionado debido al interés creativo y académico tanto de profesores como de estudiantes.

En los talleres de la Bauhaus, se comenzaron a elaborar productos funcionales y de diseño atractivo para el mercado y con bajos costos de producción; a esto le llamaron la unión del diseño industrial con la corriente artística. Se empezaron a producir objetos con materiales comunes y económicos, como el metal, el vidrio, el cristal y la madera; la demanda que se generó de más y mejores bienes impulsó a que la producción fuera en serie, los objetos concebidos por la Bauhaus contaban con un análisis de la funcionalidad para desarrollar su forma y estos debían ser pensados para una producción industrial.

Otro de los cambios importantes se dio en el año de 1929 en Dessau. Gropius realiza la tan ansiada sección de arquitectura, misma que quedó a cargo del arquitecto suizo Hannes Meyer; cuando se implementa este departamento, se reestructura todo el organigrama de la escuela logrando colocar en primera línea a la arquitectura dependiendo de ésta, lo que se denominó como "Construcción y equipamiento interior", en donde se involucraron los talleres de metal, de textil, de carpintería y de pintura mural. Más adelante incluyeron el taller de fotografía, todos los talleres tenían su área formativa, así como su área productiva.

Las reformas de Meyer no se limitaron a nivel de estructura sino también se reorientaron los principios académicos de la escuela, por ejemplo, dentro de estos principios se pedía que se contemplase el estudio de la utilidad de los objetos, la investigación de las necesidades del consumidor y la competencia de precios, con la idea de producir una serie de diseños "estándar" que fueran accesibles para la mayoría de la población. Este enfoque ha cambiado desde que las competencias académicas han permeado en el proceso de la enseñanza del Diseño; sobre todo en el aprendizaje basado en proyectos que refleja una investigación relacionada con la comunicación intercultural. 
A través de la historia se ha mencionado que La Bauhaus siempre estuvo bajo sospecha de la clase política, y que a medida que Hitler iba ganando terreno en Alemania ésta escuela se veía cada vez más amenazada en relación a su desaparición, La Bauhaus fue cerrada el 22 de agosto de 1932.

\section{El diseño como social como disciplina}

Esta investigación está dirigida al área del diseño social, y es que el proceso proyectual del diseño trae implícita de manera general la función social, ya que está considerado como una disciplina humanista, aunque no siempre se logre o se complete el objetivo marcado trazado desde el inicio del proyecto, ya sea por cuestiones de contexto geográfico, por aspectos económicos, sociales, y ambientales entre otros.

Abordar el tema de diseño social, va más allá de lo que ha marcado la historia del diseño, y que desde siempre ha tenido como principal objetivo mejorar la calidad de vida del ser humano por medio del diseño de productos con propuestas con diferentes materiales, diferentes técnicas, diferentes procesos, y que por lo general se busca el manejo de bajos costos en la producción, y es que el concepto de diseño social es un término relativamente nuevo y que va más allá de lo antes mencionado. El concepto de diseño social lo han llevado a cabo con muy poca difusión y aplicación en los contenidos de los programas académicos de las universidades de diseño.

Pérez \& Martínez (2016), ha comentado que igualmente se puede decir que el producto de diseño es un bien social en tanto su objetivo general es ser usado por varias y diversas personas, los diseños permiten experimentar formas de actuar y se convierten en parte de los entornos que soportan las actividades cotidianas. Los productos de diseño no solamente proporcionan una mayor eficacia en relación con determinadas tareas sino que permiten a los consumidores adquirir el progreso hacia formas ideales de vida y de trabajo, esta cualidad debería ser aún más relevante a la luz de los retos sociales complejos y los tipos de relaciones sociales que ellos pueden provocar y mediar.

Soto (2016) en su artículo "El rediseño del diseño", mencionó que otro de los mitos en el diseño moderno es su función social, en donde plantea que éste contribuye a mejorar las condiciones de la vida de la gente y promueve el progreso y los valores socialmente beneficiosos; pero hay que concluir que no tiene fines propios y no posee autonomía ni objetivos particulares y que es un medio de producción y no puede considerarse como una herramienta ideológica. En cuanto a su "misión", es un acto comunicacional sin pretender que tenga fines útiles y positivos para la sociedad y que deba mejorar la vida de la gente. Sintetiza mencionando que el diseño no posee una función social si nos referimos a ésta como una función humanitaria, puesto que para que actúe como tal debe existir un cliente que pide un encargo bajo unos propósitos específicos, sean estos o no axiológicamente válidos para el diseñador, lo cual no influye en el hecho de aceptar o no el trabajo.

A cinco años de haber iniciado académicamente la Licenciatura en Diseño de Artesanías en el Centro Universitario de Tonalá de la Universidad de GUADALAJARA (CUTonalá) y actualmente ya con 4 generaciones de egresados, se han llevado a cabo procesos de evaluación de procesos académicos por organismos externos a la universidad de Guadalajara, a partir de esto las autoridades del centro universitario están proyectando una reestructuración de la malla curricular misma que deberá estar enfocada específicamente hacia el diseño social. En este proyecto será importante revisar e incluir asignaturas que 
impacten en el área de las ciencias sociales, humanas y de sustentabilidad, tratando no solo de enfocarse en áreas de producción y comercialización, además del proceso proyectual del diseño como tal, sino en el o los usuarios específicos de alguna comunidad con necesidades específicas en vías de mejora de la calidad de vida. Sin embargo, el cambiar la currícula no será un proceso fácil, ni lo único que se tendrá que hacer, ya que no solo implica una serie de procedimientos burocráticos, sino que se deberán de conformar equipos de trabajo en donde no solo deberá de interactuar la academia de profesores; sino además incluir al comité consultivo mismo que está formado por varios sectores de la sociedad; este equipo de trabajo tendrá la tarea de que investigar, analizar, revisar, puntuar, normar y estandarizar, la inclusión de los elementos que intervienen en el proceso de la aplicación del diseño social plasmados en el diseño del producto con diseño artesanal.

Bastidas y col., reconocen que el Diseño Social como enfoque disciplinar adquiere cada día más reconocimiento e interés por parte de las instituciones educativas, gubernamentales, sociales, políticas y empresariales, campos de pensamiento y acción como la responsabilidad social, la innovación social, la sustentabilidad, el desarrollo humano y el cambio social enmarcan retos y oportunidades para el ejercicio del diseño con un enfoque centrado en la resolución de problemas sociales, orientado al mejoramiento de la calidad de vida, la equidad social, la humanización de la tecnología y la sostenibilidad ambiental. (Bastidas \& Martínez, 2016).

Por eso, el término de diseño social es también considerado por los investigadores como el denominador general de un área de interés disciplinar, bajo la cual se agrupan diversas dinámicas de acción del diseño que se diferencien por su especificidad en el enfoque, por el uso de herramientas, por las formas de hacer diseño o de vincular a los grupos humanos afectados. Así el término permite identificar rápidamente el ámbito y el alcance de la acción del diseño, resaltando no solo las diferencias, sino más bien lo que tienen en común: la visión, el propósito y la responsabilidad de mejorar la calidad de vida.

Uno de los principales retos y objetivos académicos es lograr coherencia y equilibrio entre la ética y la responsabilidad social y medioambiental de la profesión, con la innovación y el desarrollo tecnológico y científico. El reto para el CUTonalá será entonces, trabajar con el enfoque del aprendizaje basado en la resolución de problemas, implementado en proyectos de diseño con una carga importante de responsabilidad social.

Thackara enuncia las implicaciones del diseño consciente en donde propone analizar las consecuencias de las acciones de diseño antes de ponerlas en marcha, prestando especial atención a los sistemas naturales, industriales y culturales presentes en el contexto donde dichas acciones se realizan. Recomienda considerar qué materiales y recursos energéticos se involucran en los sistemas a diseñar, priorizar la entidad humana y no tratar a la gente como un simple "factor" dentro de algo mayor. Tratar el "contenido" como lo que hacemos, no como lo que vendemos, trabajar con el lugar, el tiempo y la diferencia cultural como valores positivos, no como obstáculos, centrarse en los servicios y no en las cosas, y abstenerse de inundar el mundo con artefactos carentes de sentido. (Thackara, 2013)

Thackara da a entender entonces que el diseño es en realidad un modo de trabajar apoyado en la idea de que la ética y la responsabilidad social pueden conformar las decisiones de diseño sin constreñir la innovación y el desarrollo tecnológico que las di- 
ferentes sociedades necesitan llevar a cabo. Por lo tanto, la responsabilidad social y la interculturalidad son importantes para el aprendizaje del Diseño.

Para Nancy Quiroga Buitrago (2014) el diseño tiene muchas facetas que interesan a los diseñadores, como, por ejemplo, las relaciones con sus clientes, los problemas conceptuales y metodológicos, los aspectos técnicos y los de la formación profesional, sin embargo, considera que el más urgente de todos es el de los efectos del diseño sobre la sociedad, el diseño contribuye a configurar nuestro entorno y hacerlo más inteligible y mejor utilizable. Los demás son problemas internos que conciernen al trabajo personal de cada diseñador, pero no a una función social.

El horizonte de acción del diseño social hoy es una cultura y forma de acción que busca el mejoramiento de las condiciones de vida y la expresión de los derechos y libertades de la sociedad. El diseño social como tal enfrenta el reto de poder lograr que se generen condiciones de vida más equitativas y globales, pero en las que se respeten y exalten las cualidades $y$ estilos de vida tanto individuales como los locales. De ahí la importancia de contar con profesionistas capacitadosy centrados en mejorar las condiciones de vida del usuario, más allá de enfocarse en el aspecto económico. El enfoque académico del aprendizaje basado en problemas aportará en los estudiantes la idea de resolver una situación que mejore la vida del usuario, partiendo del desarrollo sustentable y la responsabilidad social.

En relación a las definiciones de los expertos en diseño social, éstos coinciden en que el diseño social es una cualidad del diseño que demanda que los diseñadores sean conscientes del compromiso de pensar y actuar en proponer soluciones de manera creativa y que éstas estén dirigidas a responder a los plan- teamientos de problemas que aquejan a una comunidad, que es importante que se generen programas de acción en donde se tome en cuenta desde la insatisfacción de las necesidades físicas y emocionales, hasta la exigencia de los derechos, del grupo de usuarios, sin dejar de lado la innovación en el diseño de los productos.

Manejar el tema del diseño social en el área académica como un reto social es una cuestión compleja debido a su interdisciplinariedad en el proceso del método proyectual, para esto se deben de tomar en cuenta los efectos que puedan conformar de manera más creativa, evidente y positiva nuestro entorno, cuidar que éstos estén enfocados en propuestas de solución por medio del diseño que logren satisfacer desde las necesidades físicas, personales y emocionales, así como la expresión de la exigencia de los derechos humanos.

Los docentes en la licenciatura en diseño de artesanía de toda la malla curricular, deberán entonces de empezar a incluir en los programas educativos el tema de diseño social dentro de los objetivos de aprendizaje de la misma, para lograr que se conozca y se aplique como una cultura que busca el mejoramiento de las condiciones de vida en todas las clases sociales, partiendo del planteamiento de alguna problemática para iniciar con el análisis del propio contexto cultural, social, político, económico y ambiental, fundamentado en un enfoque centrado en la resolución de problemas sociales, con base en el mejoramiento de la calidad de vida, la equidad social, la responsabilidad en el uso de la tecnología y los aspectos de sustentabilidad ambiental, con un sentido de ética y responsabilidad social.

\section{Método proyectual de diseño}

Hablar de etapas en procesos de diseño es hablar de plantear la metodología del diseño en sí; es decir significa trabajar siguiendo las 
bases o pasos de algún método proyectual establecido, en esta ocasión esto es analizado desde el punto de vista académico para poder identificar la influencia de la comunicación intercultural en el contexto del diseño social, en el desarrollo de alguna comunidad y la relación con los demás países.

El método proyectual de diseño o metodología del diseño es una materia que marca una importante interdisciplinaridad con la mayoría de las otras materias de la malla curricular de la licenciatura de Diseño de Artesanías, esta situación invita a adentrarse en los diferentes fenómenos interculturales de otras localidades, aunque los planteamientos de los problemas de diseño sean diferentes, el objetivo general de la metodología del diseño se basa en dar soluciones de manera global.

Para iniciar el proceso de diseño es importante siempre establecer un método proyectual, o metodología, Bruno Munari (1983) en su libro como nacen los objetos menciona que no es más que una serie de pasos dictados por la experiencia y que llevados de manera secuencial nos ayudarán a trabajar mejor y con menor esfuerzo, ya que esa es la función principal para que podamos garantizar el logro de un óptimo diseño al final del proyecto.

Dentro de la "Metodología del Diseño" de Vilchis, la metodología del diseño integra un conjunto de indicaciones y prescripciones para la solución de problemas derivados del diseño, la misma metodología determina la secuencia más adecuada de acciones, su contenido y los procedimientos específicos. (Vilchis, 2002)

Pérez (2002) en su artículo "Recorrido histórico en la metodología del diseño, alude al método proyectual que Archer propuso en 1963 y que consta de listas de comprobación que determinan el proceso de diseño, pero éstas apenas serán aprovechables por su excesiva formalización. El proceso de diseño debe contener fundamentalmente las etapas de análisis, la etapa creativa y la de ejecución. A su vez estas etapas se subdividen en las siguientes fases:

- Definición del problema.

- Obtener datos, preparar especificaciones y retroalimentar la fase de la definición del problema.

- Análisis y síntesis de los datos para preparar propuestas de diseño.

- Desarrollo de prototipos.

- Preparar estudios y experimentos que validen el diseño.

- Preparar documentos para la producción.

La fundamentación de las ideas de Archer pertenecen al método científico que se maneja en los procesos de investigación cualitativa así como cuantitativa, este es el método proyectual con el que se trabaja con los estudiantes de Diseño de Artesanías en el CUTonalá, mediante proyectos en cada ciclo escolar, o bien también lo utilizan como soporte o guía del tema de investigación para la elaboración de la tesis para obtener el grado en la licenciatura.

Para los proyectos de diseño de productos artesanales en su trayecto académico, las propuestas de metodología que Archer propone son las utilizadas por los profesores, ya que es necesario presentar el diseño terminado con el proceso de principio a fin y poder ser sometido a crítica por parte de los profesores. Generando una atmosfera muy semejante a la realidad laboral próxima.

Las propuestas de metodología del diseño merecen una revisión para que se aplique de manera clara la parte del estudio y aplicación del diseño social 


\section{Método proyectual en el diseño social}

Para un proyecto de diseño de cualquier especialidad o de diseño social, tema específico de esta investigación, es importante mencionar que los métodos proyectuales no son exactos y mucho menos rigurosos, ya que ofrecen la oportunidad de revisar, analizar y corregir, es muy importante la creatividad del diseñador en el proceso de la investigación, ya que según el tipo y requisitos del proyecto de diseño, los alumnos se pueden apoyar aplicando sus propios instrumentos metodológicos siempre cuidando de no perder de vista los aspectos de la interdisciplinaridad y la problemática social real a los que deben de ofrecer una solución.

De manera específica y en relación al método proyectual del diseño social, Margolin (2015) sugiere que es importante iniciar el proceso de diseño creando una agenda de investigación amplia para abordar el tema, considerando iniciar una serie de cuestiones, como, por ejemplo: ¿Qué papel puede desempeñar un diseñador en un proceso colaborativo de intervención social? ¿Qué se está haciendo actualmente en este sentido y qué debe hacerse? ¿Cómo se puede cambiar la percepción pública de los diseñadores con el fin de presentar una imagen de un diseñador socialmente responsable? ¿Cómo pueden las organizaciones que financian investigaciones y proyectos de bienestar social tener una percepción más fuerte del diseño como una actividad socialmente responsable? ¿Qué tipos de productos satisfacen las necesidades de las poblaciones vulnerables?

Otra técnica de investigación adecuada y que aplican los diseñadores en el proceso de diseño social y que les arroja datos importantes, es la observación participante, esta etapa sitúa e involucra a los diseñadores en los diferentes contextos sociales y culturales, ya sea como parte de un equipo multidisciplinar o de manera individual para observar, analizar y docu- mentar las necesidades sociales de alguna comunidad y que por medio de propuestas de diseño puedan solucionarse.

Margolin (2015) considera que el alcance de la investigación para el diseño social incluye las percepciones públicas y de las agencias acerca de los diseñadores, la economía de las intervenciones sociales, el valor del diseño para mejorar la vida de poblaciones marginadas, una taxonomía de nuevas tipologías de producto, la economía de la fabricación de productos socialmente responsables y la forma en que dichos productos y servicios son recibidos por las poblaciones necesitadas, por lo tanto los estudiantes de diseño social aprenden más de cerca las necesidades sociales y cómo éstas son enfrentadas actualmente por los profesionales de apoyo. Se ha considerado que podrían hacer una especie de pasantía con un equipo clínico en un hospital psiquiátrico, en una agencia comunitaria o en instalaciones residenciales para ancianos. También requerirán una fundamentación más fuerte en la sociología, la psicología y la política pública. En cuanto somos conscientes, ningún programa universitario forma y entrena específicamente diseñadores sociales.

\section{El papel de la comunicación del diseño so- cial en el proceso de enseñanza}

Amarilis Elías (2014) sugiere que la universidad moderna debe estar capacitada para responder a las necesidades y los desafíos que impone la sociedad actual, estamos en tiempos de una sociedad del conocimiento y el fenómeno de la globalización, por ello en el ámbito de la formación de profesionales, es necesario lograr altos niveles de calidad en la enseñanza. Para ello es recomendable socializar el tema en equipos a fin de contribuir a generar un marco conceptual común, para todas las carreras de diseño en sus diferentes áreas de la universidad y entre las diferentes universidades latinoamericanas. 
Para esto las instituciones universitarias y responsables de las academias deben proponer líneas de investigación, que contribuyan a establecer los lineamientos para establecer como operativa la responsabilidad social y desarrollar, en cooperación con otras universidades, programas y proyectos conjuntos que permitan fijar objetivos comunes que contribuyan desde la disciplina del diseño a humanizar nuestro mundo, así como a mejorar las comunicaciones entre los seres humanos y sensibilizar a la comunidad de diseñadores sobre el tema de la responsabilidad social en Latinoamérica.

Se hace necesario que en las universidades latinoamericanas se incorpore la responsabilidad social en el currículum de las carreras y el desarrollo de un modelo metodológico, innovador, interdisciplinario, creativo, participativo y colaborativo, que integre la formación cognitiva, técnica, personal-social y moral en los estudiantes de las universidades y cooperar entre las mismas para garantizar el logro de las metas que se desean alcanzar. Al mismo tiempo propiciar, en los estudiantes y docentes, la generación de una mayor conciencia sobre la responsabilidad del alumno en su propia formación con una conducta que le da valor a la responsabilidad social, estas acciones aportan al desarrollo y evolución en la enseñanza aprendizaje desde modelos cooperativos, interdisciplinarios y de servicio a la comunidad. Actividades que se verán reforzadas en los estudios de intercambio que llevan a cabo los estudiantes de diferentes universidades en el mundo entre s.

Es importante mencionar que la demanda de la comunicación o difusión de la asignatura de diseño social en el contexto académico y global, se da gracias al avance de la tecnología, el fácil acceso que se tiene a la información y al desarrollo de la conciencia social que se ha venido desarrollando acerca del cuidado de nuestro planeta y sus recursos no renovables, por lo tanto es importante que se revisen cuidadosamente los requerimientos de los proyectos de diseño, así como el método proyectual que se tendrá que aplicar en las aulas para poder relacionarlo con las demandas sociales, y en consecuencia para modificar y relacionar los objetivos académicos con objetivos de la asignatura de metodología del diseño, no se debe olvidar que es importante incluir objetivos de formación y que estos pudiesen ser evaluados en la presentación de los proyectos de diseño por un comité académico interdisciplinario que por supuesto sean los representantes de las demás áreas del diseño.

Un aspecto muy importante de comunicación en el diseño en el proceso de enseñanza de los académicos es sobre todas las cosas dominar el manejo de la difusión en el interior de la institución así como en el exterior, de que los egresados de las licenciaturas de diseño que deciden inclinarse por el área de diseño social en cualquiera de sus especialidades no son un grupo de activistas, sino un grupo de diseñadores con fines activistas, ya que desarrollan propuestas de proyectos de diseño de productos en bien de comunidades social y económicamente poco favorecidas.

\section{La comunicación intercultural en el proceso del aprendizaje}

Para poder resaltar la importancia de la comunicación intercultural en el ámbito educativo, se recordarán primero de manera general los diferentes conceptos de cultura y comunicación de algunos autores a fin de identificar los elementos que influyen en el desarrollo de cada comunidad (Roustan, 2017).

Tal como lo propone Martha Rizo (2006), la cultura no admite una definición meramente antropológica; precisa en cambio de un abordaje simbólico que permita dar cuenta del diverso y complejo universo de sentidos con- 
tenido en ella. Por lo tanto, la cultura se basa además en aspectos sociológicos, psicológicosy de comunicación.

La cultura es un sistema de símbolos compartidos, creados por un grupo de personas que les permite manejar su medio ambiente físico, psicológico y social, ya que la cultura les proporciona un marco de referencia cognoscitivo general para una comprensión de su mundo y el funcionamiento en el mismo, esto les permite interactuar con otras personas y hacer predicciones de expectativas y acontecimientos. Las necesidades de una cultura pueden variar al igual que las prioridades relacionadas con la conducta de ciertos grupos. Una cuestión de primordial importancia en una cultura puede significar algo diferente para otra, la cultura se aprende desde el nacimiento y se transmite a las siguientes generaciones, cada generación contribuye al fondo del conocimiento y experiencia de su propia cultura para permitirle adaptarse a los nuevos retos. (Asunción Lande 1986)

Entendiendo al concepto de cultura como una serie de símbolos dentro de un contexto sociocultural que identifica a una comunidad entre sus miembros y hacia el exterior en relación con otras comunidades y por otra parte identificando el concepto de comunicación como la acción que se da gracias a la interacción entre las personas de una comunidad por medio de signos, por los cuales se transmite conocimiento, ya sea en el interior así como hacia el exterior, por lo tanto consideramos que para el proceso de enseñanza del diseño el concepto de cultura y comunicación no se pueden separar, y con esta base nos podremos adentrar en el concepto de la interculturalidad (Cordero, 2013).

La comunicación intercultural puede ayudar a crear una atmósfera que promueva la cooperación y el entendimiento entre las diferen- tes culturas, ya que representa características especiales que le pueden permitir realizar tal función, estas características son: sensibilidad a las diferencias culturales y una apreciación de la singularidad cultural; tolerancia para las conductas de comunicación ambiguas; el deseo de aceptar lo inesperado; flexibilidad para cambiar o adoptar alternativas; y expectativas reducidas respecto a una comunicación efectiva. En el CUTonalá en la licenciatura de Diseño de Artesanías, se pueden generar ambientes de cooperación y entendimiento, mediante el aprendizaje basado en proyectos que lleguen a comunidades específicas, contribuyendo con esto a una comunicación intercultural entre el estudiante de diseño y la comunidad.

Los principales objetivos del estudio de la comunicación intercultural incluyen comprender el impacto de la cultura sobre la comunicación; desarrollar la capacidad y la habilidad de percibir y considerar puntos de vista alternativos; desarrollar habilidades para realizar investigaciones en contextos interculturales y multiculturales, y para interpretar los resultados de los estudios empíricos en el área. (Pérez, J. F. C. 2004)

Los aspectos más positivos de los distintos modelos educativos de educación intercultural puestos en práctica en las últimas décadas han venido of reciendo las pistas sobre las que se debe seguir trabajando hacia esa calidad "para todas y todos". Así, los aciertos y errores, los puntos fuertes y débiles han conducido a la concepción de nuevos modelos o modelos holísticos con grandes posibilidades. Se trata de modelos donde el personal de la escuela mantiene actitudes y valores democráticos; donde se legitima la diversidad étnica y cultural; donde los procedimientos de evaluación favorecen la igualdad; donde los currículum y los materiales de enseñanza presentan las diversas perspectivas culturales; donde el 
pluralismo lingüístico y la diversidad son valorados y promovidos; donde los estilos de enseñanza y la motivación son adaptados al grupo; donde el alumnado de los diferentes grupos culturales disfrutan del mismo estatus en la escuela; donde profesorado y alumnado adquiere las habilidades y perspectivas necesarias para reconocer formas variadas de racismo y emprender acciones orientadas a su eliminación; donde los estudiantes son entrenados en la resolución positiva de los conflictos. (Pérez, J. F. C. 2004)

A raíz de lo anterior se puede apreciar como la comunicación intercultural ha marcado un avance en la generación de nuevos conocimientos, esto fomenta y motiva la necesidad de la actualización y modificación de los planes de estudio o programas educativos en la enseñanza del diseño, por lo que será importante que las autoridades analicen la posibilidad de incluir una asignatura basada en la comunicación intercultural, en donde las competencias a desarrollar en los estudiantes sean las de conocer como el proceso de comunicación intercultural contribuye en la solución de conflictos para lograr por medio del diseño elevar la calidad de vida en las comunidades y a partir de ahí, poder aplicar estos conocimientos al proyecto de diseño social tan poco abordado (Roustan, 2017).

\section{Conclusiones}

La materia de diseño, como eje del que currículo existe desde el inicio de la enseñanza del diseño como disciplina, en donde sus objetivos como se ha visto están establecidos para satisfacer las necesidades de funciones específicas de un producto y que estas necesidades van dirigidas a un grupo de usuarios que de alguna manera no siempre podrán marcar el total de una comunidad. Sin embargo, el concepto de diseño social como lo han expuesto algunos autores y lo hemos analizado, se deberá proponer de alguna manera como una asignatura específica y colocarla en semestres intermedios y avanzados, en donde los alumnos ya cuentan con el dominio de ciertos conceptos y habilidades para aplicar el aprendizaje basado en proyectos de diseño social. Es importante ser muy exigentes y minuciosos en el contenido de la calidad de los temas de proyectos académicos, es decir que se proponga de manera clara la utilidad de la multidisciplinariedad con las otras áreas del conocimiento de este currículo, con la comunidad en cuestión, así como con los participantes externos que podrían ser empresas que estén certificadas como socialmente responsables. Con esto se podría lograr que la empresa se vincule y participe para fortalecer la fundamentación de las soluciones de diseño social por parte de los estudiantes.

Para llevar a cabo esta multidisciplinariedad, deberán formar un comité o trabajar en equipos dentro de la misma universidad con otras licenciaturas de un mismo campus o centro universitario; como proyecto piloto se podría iniciar como proyecto académico de manera local, y el comité o los equipos deberán estar conformados por profesionales de las diferentes áreas del conocimiento que se caractericen por su responsabilidad y compromiso en relación al trabajo en equipo. Los comités de trabajo deberán realizar sus actividades con calidad en todos los aspectos, es decir desde el planteamiento del problema de diseño, el proceso de la conceptualización de acuerdo a las demandas de diseño en relación a la problemática planteada, la presentación de las propuestas de diseño para la revisión y análisis por cada área del conocimiento y sus posibles recomendaciones, las subsecuentes presentaciones de propuesta de diseño siguiendo las recomendaciones en secuencia emitidas por los expertos, hasta llegar a la materialización o ejecución, del proyecto.

Tratar de establecer de alguna manera dentro 
del proceso de evaluación del alumno el compromiso real con las necesidades de alguna comunidad en particular, evaluar la iniciativa, evaluar que sean cooperativos, y por supuesto es importante que sean alumnos con valores sociales, que muestren siempre el respeto a las opiniones de los demás; aquí se incluye a los usuarios de la comunidad en cuestión, al comité evaluador del proyecto del diseño y por supuesto a las empresas interesadas en las causas sociales.

"Diseño Social", este concepto va más allá de lo que conocemos como diseño sustentable, es un concepto que está muy de moda y que actualmente si se está aplicando en el área de diseño de producto, pero hablar de Diseño social va más allá, y con esto decimos que va más allá de la intención de salvar el planeta para no acabarnos los materiales naturales no renovables, va más allá de tratar de no contaminar el medio ambiente, va más allá de diseñar por medio del reciclaje o reutilización de diferentes materiales, etc. etc., sino que se trata de hablar de la verdadera función social, una función que marca una vía de comunicación y que es global, es en donde los seres humanos en el papel de usuarios juegan a ser el centro de atención dentro de una comunidad que por lo regular son de bajos niveles socioeconómicos y que se demanda la mejora de la calidad de vida simplemente como un derecho humano social en función o a partir de la disciplina del diseño.

Un aspecto muy importante de comunicación intercultural del diseño social en el proceso de enseñanza como académicos, es cuidar que siempre se manejen la difusión tanto en el interior de la institución como en el exterior de esta, dar a conocer que los egresados de la Licenciatura en Diseño de Artesanías, que se decidan inclinarse por el área de diseño social en cualquiera de sus especialidades, no son un grupo de activistas con manejo de pro- puestas altruistas. Hay que dar a conocer que los profesionales del diseño social son más que eso: que la sociedad conozca que son profesionales que se preocupan por el futuro del medio ambiente, que se enfocan en dar soluciones de manera conjunta en una propuesta de diseño, que desarrollan propuestas de proyectos de diseño de productos con calidad total en comunidades económicamente poco favorecidas, entre otros.

Para finalizar se deja como reflexión, que, como sociedad educativa en el área de la enseñanza del diseño, se tiene la importante tarea de dar cuenta que el método proyectual de un diseñador social es complejo, desde el mismo proceso de conceptualización hasta la ejecución del proyecto, y que gracias al paso de los años y al avance de aspectos tecnológicos, y de generación de conciencia social tenemos la valiosa responsabilidad de educar en este tenor. 


\section{Referencias}

Asunción-Lande, N. (1986). Comunicación intercultural. Comunicación humana ciencia social, México: Mc Graw-Hill, 179-193.

Bastidas, A., \& Martínez, H. R. (2016). Diseño social: Tendencias, enfoques y campos de acción. Arquetipo, (13), 89-113.

Buitrago, N. Q. (2014). Diseño con proyección social: Sinergia entre la academia y los procesos comunitarios.

Bruno, M. (1989). ¿Cómo nacen los objetos? GG Diseño.

Cordero, I. (2013). Didáctica y enseñanza del Derecho en las universidades. Pensamiento Americano. 6(11) 29-34

Cembellín, B. H. (2004). Bauhaus, la escuela que unió arte y técnica. Técnica industrial, (252), 68-75.

Elías, A. (2010). La Enseñanza y responsabilidad social en el diseño. Actas de Diseño. Facultad de Diseño y Comunicación. Universidad de Palermo. ISSN, 1850, 2032.

Margolin, V. (2015). Un "modelo social" de diseño: cuestiones de práctica e investigación. Revista Kepes, 61-71.

Munari, B., \& Rodriguez, C. A. (1983). ¿Cómo nacen los objetos? GG.

Pérez, J. F. C. (2004). Interculturalidad y educación. Educatio siglo XXI, 22, 9-17.

Pérez,A. B., \&Martínez, H. R. (2016). DiseñoSocial: Enfoques y proyectos de diseño industrial en la Universidad Autónoma de Colombia. InvestigiumIRE, 7(2), 4864.

Pérez, J. F., Pujades, N. V., Picas, J. T., \& Orus, X. E. (2002). Recorrido Histórico en la metodología del diseño. In Santander, XIV Congreso Internacional de Ingeniería Gráfica, Universidad de Cantabria.

Rizo, M., \& Romeu, V. (2006). Cultura y comunicación intercultural. Aproximaciones conceptuales. Revista da Associação Nacional dos Programas de Pós-Graduação em Comunicação, 6.

Roustan, M. (2017). Análisis de la comunicación grupal: un año de experiencia con un grupo terapéutico de ayudainteractiva.PensamientoAmericano,10(18),2130. http://dx.doi.org/10.21803\%2Fpenamer.10.18.384

Scott Robert, G. I. L. L. A. M. (1969). Fundamentos del diseño. La Habana, Edición Revolucionaria.

Soto, C. (2016) Ponencia Pedagogía del Diseño: una responsabilidad Social en el II Congreso Latinoamericano de la Enseñanza del Diseño en la
Universidad de Palermo, Buenos Aires, Argentina.

Vargas, E. C., \& Oguri, L. E. (2013). El arte-objeto ¿es diseño industrial? Revista Legado de Arquitectura y Diseño, (14), 9-20.

Vilchis, L. (2002). Metodología del diseño: fundamentos teóricos. UNAM.

Thackara, J. (2013). Diseñando para un mundo complejo: acciones para lograr la sustentabilidad. DR Editorial Designio.

Wong, W. (1995). Fundamentos del diseño. Gustavo Gili. 